\title{
Convalescent plasma and hyperimmune immunoglobulin to prevent infection with SARS-CoV-2 (Protocol)
}

Valk SJ, Piechotta V, Kimber C, Chai KL, Monsef I, Doree C, Wood EM, Lamikanra AA, Roberts DJ, McQuilten Z, So-Osman C, Estcourt LJ, Skoetz N

Valk SJ, Piechotta V, Kimber C, Chai KL, Monsef I, Doree C, Wood EM, Lamikanra AA, Roberts DJ, McQuilten Z, So-Osman C, Estcourt LJ, Skoetz N.

Convalescent plasma and hyperimmune immunoglobulin to prevent infection with SARS-CoV-2 (Protocol).

Cochrane Database of Systematic Reviews 2020, Issue 12. Art. No.: CD013802.

DOI: 10.1002/14651858.CD013802.

www.cochranelibrary.com 
TABLE OF CONTENTS

HEADER 1

ABSTRACT

BACKGROUND

OBJECTIVES

METHODS

ACKNOWLEDGEMENTS

REFERENCES

APPENDICES

HISTORY

CONTRIBUTIONS OF AUTHORS

DECLARATIONS OF INTEREST

SOURCES OF SUPPORT 
[Intervention Protocol]

\section{Convalescent plasma and hyperimmune immunoglobulin to prevent infection with SARS-CoV-2}

Sarah J Valk1,2a, Vanessa Piechotta ${ }^{3 b}$, Catherine Kimber ${ }^{4}$, Khai Li Chai ${ }^{5}$, Ina Monsef ${ }^{3}$, Carolyn Doree ${ }^{4}$, Erica M Wood ${ }^{5}$, Abigail A Lamikanra6, David J Roberts $^{4}$, Zoe McQuilten ${ }^{5}$, Cynthia So-Osman 7,8 , Lise J Estcourt ${ }^{9}$, Nicole Skoetz ${ }^{10}$

1Jon J van Rood Center for Clinical Transfusion Research, Sanquin/Leiden University Medical Center, Leiden, Netherlands. 2Department of Clinical Epidemiology, Leiden University Medical Center, Leiden, Netherlands. ${ }^{3}$ Cochrane Haematology, Department I of Internal Medicine, Center for Integrated Oncology Aachen Bonn Cologne Duesseldorf, Faculty of Medicine and University Hospital Cologne, University of Cologne, Cologne, Germany. ${ }^{4}$ Systematic Review Initiative, NHS Blood and Transplant, Oxford, UK. ${ }^{5}$ Transfusion Research Unit, School of Public Health and Preventive Medicine, Monash University, Melbourne, Australia. ${ }^{6}$ Clinical, Research and Development, NHS Blood and Transplant, Oxford, UK. ${ }^{7}$ Erasmus Medical Centre, Rotterdam, Netherlands. ${ }^{8}$ Sanquin Blood Bank, Amsterdam, Netherlands. ${ }^{9}$ Haematology/Transfusion Medicine, NHS Blood and Transplant, Oxford, UK. ${ }^{10}$ Cochrane Cancer, Department I of Internal Medicine, Center for Integrated Oncology Aachen Bonn Cologne Duesseldorf, Faculty of Medicine and University Hospital Cologne, University of Cologne, Cologne, Germany

$a_{\text {contributed equally. }} b_{\text {contributed equally }}$

Contact address: Lise J Estcourt, lise.estcourt@nhsbt.nhs.uk, lise.estcourt@ndcls.ox.ac.uk.

Editorial group: Cochrane Haematology Group.

Publication status and date: New, published in Issue 12, 2020.

Citation: Valk SJ, Piechotta V, Kimber C, Chai KL, Monsef I, Doree C, Wood EM, Lamikanra AA, Roberts DJ, McQuilten Z, So-Osman C, Estcourt LJ, Skoetz N. Convalescent plasma and hyperimmune immunoglobulin to prevent infection with SARS-CoV-2 (Protocol). Cochrane Database of Systematic Reviews 2020, Issue 12. Art. No.: CD013802. DOI: 10.1002/14651858.CD013802.

Copyright @ 2020 The Cochrane Collaboration. Published by John Wiley \& Sons, Ltd.

\section{A B S T R A C T}

\section{Objectives}

This is a protocol for a Cochrane Review (intervention). The objectives are as follows:

To assess whether convalescent plasma or hyperimmune immunoglobulin transfusion is effective and safe to prevent infection with SARSCoV-2 and development of COVID-19; and to maintain the currency of the evidence, using a living systematic review approach. 


\section{B A C K G R O U N D}

\section{Description of the condition}

The clinical syndrome coronavirus disease 2019 (COVID-19) is a new, rapidly emerging zoonotic infectious disease caused by severe acute respiratory syndrome coronavirus 2 (SARS-CoV-2; WHO 2020a). On 11 March 2020, the World Health Organization (WHO) declared the COVID-19 outbreak a pandemic, with the virus spreading rapidly in the first few months and a second wave of infections now occurring in many parts of the world (WHO 2020b; WHO 2020c). Although there are similarities with historic coronavirus epidemics, with severe acute respiratory syndrome (SARS) and Middle East respiratory syndrome (MERS) responsible for 813 and 858 deaths respectively, the scale and impact of the COVID-19 pandemic presents unprecedented challenges to health facilities and healthcare workers all over the world (WHO 2007; WHO 2019).

The hospitalisation rate for COVID-19 in the period 1 to 28 March was estimated to be 4.6 per 100,000 population in the USA (Garg 2020). Groups at particular high risk of hospitalisation were those aged 65 and above, and those with underlying medical conditions (Garg 2020). Early reports of the case fatality rate for COVID-19 suggested that it ranged between $0.7 \%$ and $4 \%$, with higher rates also reported (WHO 2020a; WHO 2020d). However, these numbers should be interpreted with great care due to the data pertaining to the early emergency response, which due to shortage of test kits, led to selective testing of people with severe disease, underreporting of cases and delays from confirmation of a case to time of death (Kim 2020). By studying the population that was quarantined on the Diamond Princess cruise ship in February 2020 , researchers have been able to generate useful insight into the case fatality rate independent of the above mentioned factors. Here, the case fatality rate taking into account the delay from confirmation to death, was shown to be $2.6 \%$ (Russell 2020). A larger study, which also employed delay-adjusted methods but performed a random-effects meta-analysis across 107 countries and the Diamond Princess cruise ship, found a case fatality rate of 2.8\% (95\% confidence interval (Cl) 2.1 to 3.1; Canelo-Aybar 2020). Age, ethnicity, access to health care, socioeconomic status and underlying health conditions have been identified as risk factors for COVID-19 mortality (Williamson 2020).

The median incubation period of SARS-CoV-2 was reported to be five days, with $97.5 \%$ of cases developing symptoms within 11.5 days of infection (Lauer 2020). Common signs and symptoms can include fever, dry cough, fatigue and sputum production (WHO 2020a). Other, less commonly reported signs and symptoms are shortness of breath, sore throat, headache, myalgia or arthralgia, chills, nausea or vomiting, nasal congestion, diarrhoea, haemoptysis and conjunctival congestion (WHO 2020a). Of the reported cases, $80 \%$ are estimated to have a mild or asymptomatic course of infection, and an estimated $5 \%$ of cases are admitted to the intensive care unit (ICU) with acute respiratory distress syndrome (ARDS), septic shock or multiple organ failure, or a combination of these (Team NCPERE 2020; WHO 2020a). A risk factor for developing infection and progressing to severe disease is old age (Wu 2020a), with people aged over 80 years at highest risk of mortality. Other risk factors are cardiovascular disease, obesity, hypertension, diabetes, and chronic respiratory disease (Chen L 2020; Huang 2020; WHO 2020a). Recent reports have suggested that people who are immune-compromised may not have an increased risk of being hospitalised with severe COVID-19 symptoms (D'Antiga 2020). However, evidence has been conflicting, with patients with malignancy and solid organ transplant recipients reported to potentially have an increased risk of severe COVID-19 disease (Fung 2020; Liang 2020). Strategies to prevent COVID-19 severe disease in these risk groups are being developed. Among these strategies is the possibility of using interventions for prophylaxis. The first study published on using hydroxychloroquine for post-exposure prophylaxis found no benefit of its use (Boulware 2020). Other studies investigating interventions aimed at preventing severe disease in the aforementioned risk groups are currently being performed.

SARS-CoV-2 is a positive-sense, single-stranded RNA (ribonucleic acid) virus with a large genome (Wu 2020b). Although not much is known about the specific mechanisms underlying severe disease in COVID-19, there are indications that the virus is capable of inducing an excessive immune reaction in the host, with highly activated but decreased numbers of $\mathrm{CD}^{+}$and $\mathrm{CD} 8^{+} \mathrm{T}$ cells detected in the peripheral blood of people suffering from severe COVID-19 (Chen Z 2020; Xu 2020). Early reports also showed that people critically ill with COVID-19 frequently exhibit a hypercoagulable state and endothelial inflammation, which is hypothesised to lead to the high burden of thromboembolic events seen in this population (Driggin 2020). Preliminary reports into the pathophysiology of SARS-CoV-2 have further indicated that the observed decrease in human angiotensin-converting enzyme 2 (ACE2) activity may play a role in causing the rapid deterioration of patient lung function (Tolouian 2020; Van de Veerdonk 2020). ACE2 is a protein that functions as the receptor facilitating entry of SARS-CoV-2 into the host cell, and is most abundant on type II alveolar cells in the lungs (Zou 2020).

\section{Description of the intervention}

Convalescent plasma, convalescent serum and hyperimmune immunoglobulin are prepared from blood donated by people who have recovered from a viral illness. Convalescent plasma has been used in the past to treat or prevent conditions where no vaccine or pharmacological interventions were available. They have been used as pre-exposure prophylaxis, as post-exposure prophylaxis and as treatment. Diphtheria, pneumococcal pneumonia, hepatitis $\mathrm{A}$ and $\mathrm{B}$, mumps, polio, measles and rabies are conditions where convalescent plasma has been shown to be effective (Eibl 2008).

Passive antibody transfer is still part of current clinical practice. For example, antibody administration is used to prevent viral disease after exposure in people exposed to hepatitis $\mathrm{B}$ or rabies viruses (CDC 2020a; CDC 2020b). Infants at high risk of severe respiratory syncytial virus (RSV) are also given passive antibody treatment which, until recently, was derived from donors and is now also available as a monoclonal antibody (Soto 2020). A double-blind, placebo-controlled study of RSV-IVIG $(750 \mathrm{mg} /$ kg every 30 days), conducted in 510 infants and children with bronchopulmonary dysplasia or prematurity without lung disease, showed it decreased hospitalisations due to RSV from $13.5 \%$ to $8.0 \%$ ( $P=0.047$; PREVENT 1997). It is recommended that pregnant women and infants exposed to varicella zoster receive varicella zoster immunoglobulin to prevent or attenuate infection (Ogilvie 1998).

Although convalescent plasma is generally thought to be a safe and well-tolerated therapy, adverse events can occur. Limited 
information is available about specific adverse events related to convalescent plasma therapy, but symptoms that have been reported are similar to those for other types of plasma blood components, including fever or chills, allergic reactions, and transfusion-related acute lung injury (TRALI; Beigel 2019; Chun 2016; Luke 2006). Furthermore, the transfer of coagulation factors present in plasma products is potentially harmful for people with COVID-19, who are already at an increased risk of thromboembolic events (Driggin 2020). Plasma transfusions are also known to cause transfusion-associated circulatory overload (TACO). TACO and TRALI are especially important to consider, because COVID-19 patients with co-morbidities, who might be eligible for experimental treatment with convalescent plasma therapy, are at an increased risk of these adverse events. There are risk-mitigation strategies that can be implemented to prevent TRALI. These include limiting donations from female donors, especially those with a history of pregnancy, and screening of donors for antibodies that are implicated in TRALI (Otrock 2017). In addition to the aforementioned adverse events, transfusion-transmitted infections, red blood cell alloimmunisation and haemolytic transfusion reactions have also been described following plasma transfusion, although they are less common (Pandey 2012). Pathogen inactivation can be implemented to decrease the risk of transmitting infections by transfusion (Rock 2011).

Safety of convalescent plasma for treatment of COVID-19 has recently been investigated in a large cohort from the US FDA (United States Food and Drug Administration) expanded access program (US Covid Plasma 2020). Here, convalescent plasma did not clearly cause an excessive risk of adverse events within seven days of treatment, nor did it show an exceptionally high mortality (8.6\%) at seven days (US Covid Plasma 2020). It is important to note that the study population consisted of patients hospitalised with severe or life-threatening COVID-19, and the safety metrics can be different in the population eligible for convalescent plasma for prophylaxis.

When compared to convalescent plasma, hyperimmune immunoglobulin has the advantage of preventing transfer of potentially harmful coagulation factors that are present in plasma products. The amount and antibody concentration can be more accurately dosed compared to convalescent plasma, and hyperimmune immunoglobulin can be prepared in a consistent manner (Hung 2013). Not many studies have reported on adverse events of hyperimmune immunoglobulin, but the safety profile of standard intravenous immunoglobulin is known and the adverse events reported here are also likely to occur in hyperimmune immunoglobulin therapy. Common adverse events of intravenous immunoglobulin that occur immediately after administration are: infusion site pain; swelling and erythema; and immediate systemic reactions, such as head and body aches, chills and fever (Stiehm 2013). Other, less common early adverse reactions to immunoglobulin therapy are pulmonary complications, such as pulmonary embolism, pulmonary oedema and pleural effusion, with TRALI also reported (Baudel 2020; Stiehm 2013). Anaphylactic and anaphylactoid reactions to immunoglobulin therapy are rare (Brennan 2003; Stiehm 2013). Delayed adverse events of immunoglobulin therapy, which occur within hours to days of initiation of immunoglobulin therapy, are persistent headaches (common), aseptic meningitis, renal failure, thromboembolic events, and haemolytic reactions (Sekul 1994; Stiehm 2013). Transmission of infectious agents has been described after administration of intravenous immunoglobulin, but this risk is considered to be low (Stiehm 2013). Other, severe adverse events that occur late after administration are lung disease, enteritis and dermatological disorders (Stiehm 2013).

A theoretical risk related to virus-specific antibodies, which are transferred with convalescent plasma and hyperimmune immunoglobulin administration, is antibodydependent enhancement of infection (Morens 1994). Here, virusbinding antibodies facilitate the entry and replication of virus particles into monocytes, macrophages and granulocytic cells and thereby increase the risk of more severe disease in the infected host. Although antibody-dependent enhancement has not been demonstrated in COVID-19, it has been seen with previous coronavirus infections when the antibodies given targeted a different serotype of the virus (Wan 2020; Wang 2014).

\section{How the intervention might work}

Convalescent plasma contains pathogen-specific neutralising antibodies, which can neutralise viral particles, and treatment with convalescent plasma or hyperimmune immunoglobulin confers passive immunity to recipients. The duration of conferred protection can differ depending on the timing of administration, ranging from weeks to months after treatment (Casadevall 2020).

By neutralising SARS-CoV-2 particles, early treatment with convalescent plasma is postulated to increase the patient's own capacity to clear the initial inoculum (Casadevall 2020; Robbins 1995). This could lead to a reduction in mortality and fewer hospitalised patients progressing to the ICU. Furthermore, convalescent plasma may reduce the length of ICU stay in critically ill patients (Mair-Jenkins 2015), thus helping to lift pressure from global healthcare systems and increasing ICU capacity.

Preliminary evidence in humans and rhesus macaques has shown that primary SARS-CoV-2 infection is capable of inducing a protective immune response, with most (but not all) patients who have recovered from COVID-19 producing sufficient amounts of neutralising antibodies to protect against reinfection (Bao 2020; Wu 2020a). This implies that convalescent plasma from people who have recovered from SARS-CoV-2 infection is capable of conferring passive immunity. A recently reported case series also indicated sufficient neutralising antibody titres in convalescent plasma to neutralise SARS-CoV-2 in five COVID-19 patients, who all recovered after treatment (Shen 2020). It is important to note, however, that research in other coronavirus species has shown that immunity may not be long-lasting, with two to three years of protection estimated from work with SARS and MERS (Mo 2006; Payne 2016). This short duration of protection has recently been demonstrated in convalescent COVID-19 patients who experienced mild illness, with antibody levels reported to decrease after two to three months, indicating the window for convalescent plasma collection may be quite small (Ibarrondo 2020). Furthermore, there are indications that the severity of infection has an impact on antibody titres, with less severe disease leading to lower neutralising antibody response in people with SARS and COVID-19 (Zhao 2020). And, it is unclear exactly how often reinfection occurs, with the burden of reinfection likely to be underestimated, while at the same time a number of case reports of severe reinfection have been published (Iwasaki 2020). 


\section{Why it is important to do this review}

With the numbers of COVID-19 cases still rising globally, the need for interventions to prevent severe COVID-19 disease remains. Pharmacological treatment options are being investigated in many ongoing studies, with currently only treatment of dexamethasone proven to be effective in reducing mortality (Horby 2020), and remdesivir shown to reduce time to recovery (Beigel 2020). Current treatment further consists of supportive care with extracorporeal membrane oxygenation in severe cases and oxygen supply in mild cases (CDC 2020c; WHO 2020e). Despite these treatments, people hospitalised with COVID-19 are still at a high risk of mortality. A vaccine could aid in inducing immunity in the population and preventing transmission to those who are at risk for severe disease, but no vaccine is currently available, although multiple candidate vaccines are in development. Until these vaccines are available and distributed, convalescent plasma is a potential prophylactic strategy for people at high risk for infection with SARS-CoV-2, or severe symptoms of COVID-19. It is important to note that even with future possibility of an effective vaccine, there could still be a use for COVID-19 prophylactic strategies, like convalescent plasma or hyperimmune immunoglobulin, if they are found effective and safe. Not everyone can be effectively vaccinated, for example, people who are temporarily or permanently immune-compromised, and very young children.

Convalescent plasma, and hyperimmune immunoglobulin to a certain extent, can be prepared and made rapidly available by blood banks and hospitals when enough potential donors have recovered from the infection, using readily available materials and methods (Bloch 2020). Recently, safety of convalescent plasma for COVID-19 has been investigated in a large observational cohort, and the numbers of adverse events and mortality at seven days were deemed to be low (US Covid Plasma 2020). There is no information available on safety of hyperimmune immunoglobulin for COVID-19. The efficacy of convalescent plasma and hyperimmune immunoglobulin is not yet well characterised, with few studies only currently having been published, which suffer from considerable methodological limitations (Chai 2020). The costs associated with pursuing the use of convalescent plasma for prophylaxis for COVID-19 should also be taken into account, especially when considering widespread implementation.

A multitude of clinical studies investigating the safety and effectiveness of convalescent plasma or hyperimmune immunoglobulin have been announced. Most of these studies include patients who are hospitalised with severe COVID-19. However, some of these studies plan to include people who will receive convalescent plasma or hyperimmune immunoglobulin for prophylaxis, either as the total study population or as a subgroup of a larger study. In summary, the benefits of the intervention, both for convalescent plasma or hyperimmune immunoglobulin, should be carefully considered in view of the risks of adverse events. This is especially important when the intervention is targeted at people who are in poor health, but are not, or not yet infected with SARSCoV-2, or do not, or not yet, experience symptoms of COVID-19.

\section{O B JE C T IVES}

To assess whether convalescent plasma or hyperimmune immunoglobulin transfusion is effective and safe to prevent infection with SARS-CoV-2 and development of COVID-19; and to maintain the currency of the evidence, using a living systematic review approach.

\section{METHODS}

\section{Criteria for considering studies for this review}

\section{Types of studies}

To assess the effectiveness and safety of convalescent plasma to prevent infection with SARS-CoV-2 we will include randomised controlled trials (RCTs), as such studies, if performed appropriately, give the best evidence for experimental therapies in highly controlled therapeutic settings. For RCT data, we will use the methods recommended by the Cochrane Handbook for Systematic Reviews of Interventions (Higgins 2020a). We will include nonstandard RCT designs, such as cluster-randomised trials and cross-over trials, and will use the methods recommended in Chapter 23 of the Cochrane Handbook for Systematic Reviews of Interventions (Higgins 2020b). We will only consider the results from the first cycle of cross-over RCTs.

We will include full-text publications, pre-print articles, abstract publications, and results published in trials registries, if sufficient information is available on study design, characteristics of participants, interventions and outcomes. We will not apply any limitation with respect to the length of follow-up.

\section{Types of participants}

We will include individuals who do not have SARS-CoV-2 but are at high risk of developing the infection (e.g. high-risk exposure) or at high risk of fatal disease (e.g. high-risk populations), with no age, gender or ethnicity restrictions.

We will exclude studies including populations with exposure to other coronavirus diseases (SARS or MERS). We will also exclude studies including populations with exposure to mixed viral diseases (e.g. influenza), unless the study authors provided subgroup data for people exposed to SARS-CoV-2.

Treatment of individuals who have SARS-CoV-2 infection with convalescent plasma or hyperimmune immunoglobulin is covered in another review (Chai 2020).

\section{Types of interventions}

We will include the following interventions.

- Convalescent plasma from people who had recovered from SARS-CoV-2 infection

- Hyperimmune immunoglobulin therapy

We will not include studies on standard immunoglobulin.

We will include the following comparisons for studies with a control arm.

- Convalescent plasma therapy versus control intervention, for example, vaccinations, standard immunoglobulin, non-immune plasma, other prevention strategies (e.g. protective clothing, face masks, social distancing), pharmaceutical interventions (e.g. hydroxychloroquine), complementary medicine (e.g. quercetin, elderberry, zinc). Co-interventions are allowed, but must be comparable between intervention groups. 
- Convalescent plasma versus no treatment or placebo

- Convalescent plasma therapy versus hyperimmune immunoglobulin

- Hyperimmune immunoglobulin versus no treatment or placebo

- Hyperimmune immunoglobulin versus control intervention, for example, vaccinations, standard immunoglobulin, non-immune plasma, other prevention strategies (e.g. protective clothing, face masks, social distancing), pharmaceutical interventions (e.g. hydroxychloroquine), complementary medicine (e.g. quercetin, elderberry, zinc). Co-interventions are allowed, but must be comparable between intervention groups.

\section{Types of outcome measures}

We will evaluate core outcomes as pre-defined by the Core Outcome Set for studies evaluating public health, primary and secondary care interventions for prevention of COVID-19 transmission (COMET 2020). The study to define outcomes is still ongoing (study completion was planned for September 2020). The below-listed outcomes are therefore subject to change.

\section{Primary outcomes}

Effectiveness of convalescent plasma or hyperimmune immunoglobulin to prevent infection with SARS-CoV-2

- Infection with SARS-CoV-2 (confirmed by positive reverse transcription polymerase chain reaction (RT-PCR) test) within 30 days

- Development of clinical COVID-19 symptoms, assessed with the WHO Clinical Progression Scale (WHO 2020f), within 30 days:

* uninfected

* ambulatory mild disease

* hospitalised with moderate disease

* hospitalised with severe disease

* death

\section{Secondary outcomes}

\section{Effectiveness of convalescent plasma or hyperimmune} immunoglobulin to prevent infection with SARS-CoV-2

- All-cause mortality until longest follow-up

- Mortality (time to event)

- Admission to hospital within 30 days

- Admission to the ICU within 30 days

- Quality of life, assessed with standardised scales (e.g. WHOQOL-100) at up to seven days; up to 30 days, and longest follow-up available

\section{Safety of convalescent plasma or hyperimmune immunoglobulin to} prevent infection with SARS-CoV-2

- Number of participants with adverse events, including potential relationship between intervention and adverse reaction (e.g. transfusion-related acute lung injury (TRALI), transfusiontransmitted infection, transfusion-associated circulatory overload (TACO), transfusion-associated dyspnoea (TAD), acute transfusion reactions)

- Number of participants with serious adverse events, including potential relationship between intervention and adverse reaction (e.g. TRALI, TACO, TAD, acute transfusion reactions)

\section{Timing of outcome measurement}

For time-to-event outcomes, such as mortality (time to event), we will include outcome measures representing the longest follow-up time available.

We will include all other outcome categories for the observational periods that the study publications report. We will include those adverse events occurring during active treatment and will include long-term adverse events as well. If sufficient data are available, we will group the measurement time points of eligible outcomes, for example, adverse events and serious adverse events, into those measured directly after treatment (up to 7 days after treatment), medium-term outcomes ( 15 days after treatment) and longer-term outcomes (over 30 days after treatment).

\section{Search methods for identification of studies}

This review is part of a COVID-19 living systematic review project evaluating effectiveness and safety of convalescent plasma and hyperimmune immunoglobulin. Our search addresses:

- convalescent plasma and hyperimmune immunoglobulin to prevent infection with SARS-CoV-2; and

- convalescent plasma and hyperimmune immunoglobulin to treat people with COVID-19 (Chai 2020).

We are carrying out weekly searches for completed and ongoing studies in all languages in order to limit language bias. We will review search methods and strategies approximately monthly, to ensure they reflect any terminology changes in the topic area, or in the databases.

\section{Electronic searches}

We designed and tested search strategies for electronic databases according to methods suggested in the Cochrane Handbook for Systematic Reviews of Interventions (Lefebvre 2020), CD developed them and Cochrane Haematology's Information Specialist (IM) peer reviewed them. In this emerging field, we expected that the abstract, at least, would be in English. If studies are published in languages other than those our review team can accommodate (English, Dutch, German, French, Italian, Malay and Spanish), we will involve Cochrane TaskExchange to identify people within Cochrane to translate these studies.

As publication bias might influence all subsequent analyses and conclusions, we will search all potentially relevant trials registries in detail to detect ongoing as well as completed studies, but not yet published studies. Nowadays, it is mandatory to provide results at least in the trials registry. In the event that these results are not published elsewhere, we plan to extract and analyse these data.

We will search the following databases and sources, starting from 1 January 2019. 
- Databases of medical literature

* MEDLINE (via Ovid), Appendix 1

* Embase (via Ovid), Appendix 2

* PubMed (for epublications ahead of print only), Appendix 3

* World Health Organization COVID-19 Global literature on coronavirus disease (search.bvsalud.org/global-literatureon-novel-coronavirus-2019-ncov), Appendix 4

* Epistemonikos, LOVE List Coronavirus disease (COVID-19) (app.iloveevidence.com), Appendix 5

* Cochrane COVID-19 Study Register (covid-19.cochrane.org), Appendix 6

- Trials registries and registry platforms to identify ongoing studies and results of completed studies

* ClinicalTrials.gov - COVID-19 subset (included in Cochrane COVID-19 Study Register)

* WHO International Clinical Trials Registry Platform (ICTRP) - COVID-19 subset (included in Cochrane COVID-19 Study Register)

* medRxiv (included in Cochrane COVID-19 Study Register)

\section{Searching other resources}

We will handsearch the reference lists of all identified studies, relevant review articles and current treatment guidelines for further literature and contact experts in the field, drug manufacturers and regulatory agencies in order to retrieve information on unpublished studies.

\section{Data collection and analysis}

\section{Selection of studies}

Two out of five review authors (SJV, KLC, VP, CK, NS) will independently screen the results of the search strategies for eligibility for this review by reading the abstracts using Covidence software (Covidence). We will immediately screen any new citations retrieved by the weekly searches and code the abstracts as either 'retrieve' or 'do not retrieve'. In the case of disagreement or if it is unclear whether we should retrieve the abstract or not, we will obtain the full-text publication for further discussion. Two review authors will assess the full-text articles of selected studies. If the two review authors are unable to reach a consensus, we will consult all review authors who are involved in the study selection to reach a final decision.

We will document the study selection process in a flow chart, as recommended in the PRISMA statement (Moher 2009), and show the total numbers of retrieved references and the numbers of included and excluded studies. We will list all articles that we excluded after full-text assessment and the reasons for their exclusion in the 'Characteristics of excluded studies' table.

\section{Data extraction and management}

We will conduct data extraction according to the guidelines proposed by Cochrane (Li 2020), using a customised data extraction form developed in Microsoft Excel (Microsoft Corporation 2018). One review author (NS, SJV, KLC, CK or VP) will perform all data extractions. Two other review authors (NS, SJV, KLC, CK or VP) will verify the accuracy and (where applicable) the plausibility of extractions.
Two review authors (SJV, KLC, VP, CK, or NS) will independently assess eligible studies obtained in the process of study selection (as described above) for methodological quality and risk of bias. If the review authors are unable to reach a consensus, we will consult a third review author).

We will collate multiple reports of one study so that the study, and not the report, is the unit of analysis.

We will extract the following information.

- General information: author, title, source, publication date, country, language, duplicate publications, funding or sponsor

- 'Risk of bias' assessment: study design, confounding, definition of risk estimates, selection bias, attrition bias, detection bias, reporting bias

- Study characteristics: study design, setting and dates, source of participants, inclusion/exclusion criteria, comparability of groups, treatment cross-overs, compliance with assigned treatment, length of follow-up

- Participant characteristics: age, gender, ethnicity, number of participants recruited/allocated/evaluated, additional diagnoses, location of exposure, concomitant prevention strategies (e.g. protective clothing, face masks, social distancing, pharmaceutical interventions (e.g. hydroxychloroquine), complementary medicine) complementary medicine (e.g. quercetin, elderberry, zinc), whether the donors were tested by nasal swabs or whether the plasma was tested

- Interventions: convalescent plasma therapy or hyperimmune immunoglobulin therapy, concomitant prevention strategies, duration of follow-up, donors' disease severity, how donations were tested for neutralising antibody

- For studies including a control group: control intervention, concomitant prevention strategies, duration of follow-up, donors' disease severity, how donations were tested for neutralising antibody

- Outcomes

* Effectiveness of convalescent plasma or hyperimmune immunoglobulin to prevent infection with SARS-Cov-2

$\square$ Infection with SARS-CoV-2 (positive RT-PCR test) within 30 days

Development of clinical COVID-19 symptoms, assessed with the WHO Clinical Progression Scale (WHO 2020f) within 30 days

$\square$ All-cause mortality until longest follow-up

Mortality (time to event)

Admission to the hospital within 30 days

Admission to the ICU within 30 days

$\square$ Quality of life, assessed with standardised scales (e.g. WHOQOL-100) at up to seven days; up to 30 days, and longest follow-up available

* Safety of convalescent plasma or hyperimmune immunoglobulin to prevent infection with SARS-Cov-2

$\square$ Number of participants with adverse events, including potential relationship between intervention and adverse reaction (e.g. TRALI, TACO, TAD, acute transfusion reactions)

Number of participants with serious adverse events, including potential relationship between 
intervention and adverse reaction (e.g. TRALI, TACO, TAD, acute transfusion reactions)

\section{Assessment of risk of bias in included studies}

We will use the Risk of Bias 2.0 (RoB 2) tool to analyse the risk of bias in the underlying study results (Sterne 2019). Of interest for this review is the effect of the assignment to the intervention (the intention-to-treat (ITT) effect) and we will perform all assessments with RoB 2 on this effect. The outcomes that we will address are those specified for inclusion in the 'Summary of findings' table. Accordingly, the outcomes will be prioritised according to the Core Outcome Set for studies evaluating public health, primary and secondary care interventions for prevention of COVID-19 transmission (COMET 2020).

Two out of five review authors (SJV, KLC, VP, CK, NS) will independently assess the risk of bias for each study result. In case of discrepancies among their judgements or inability to reach consensus, we will consult a third review author to reach a final decision. We will assess the following types of bias as outlined in Chapter 8 of the Cochrane Handbook for Systematic Reviews of Interventions (Higgins 2020c).

- Bias arising from the randomisation process

- Bias due to deviations from the intended interventions

- Bias due to missing outcome data

- Bias in measurement of the outcome

- Bias in selection of the reported result

For cluster-RCTs, we will add an additional domain to assess bias arising from the timing of identification and recruitment of participants in relation to timing of randomisation, as recommended in the archived RoB 2 guidance for clusterrandomised trials (Eldridge 2016), and in Chapter 23 of the Cochrane Handbook for Systematic Reviews of Interventions (Higgins 2020b).

For cross-over RCTs, we will not add any additional considerations, because we will only consider the results of the first cycle.

To address these types of bias we will use the signalling questions recommended in RoB 2 and make a judgement using the following options:

- 'yes': if there is firm evidence that the question is fulfilled in the study (i.e. the study is at low or high risk of bias given the direction of the question);

- 'probably yes': a judgement has been made that the question is fulfilled in the study (i.e. the study is at low or high risk of bias given the direction of the question);

- 'no': if there is firm evidence that the question is unfilled in the study (i.e. the study is at low or high risk of bias given the direction of the question);

- 'probably no': a judgement has been made that the question is unfilled in the study (i.e. the study is at low or high risk of bias given the direction of the question);

- 'no information' if the study report does not provide sufficient information to allow any judgement.

We will use the algorithms proposed by RoB 2 to assign each domain one of the following levels of bias:
- low risk of bias;

- some concerns;

- high risk of bias.

Subsequently we will derive a 'Risk of bias' rating for each prespecified outcome in each study in accordance with the following suggestions.

- 'Low risk of bias': the study is judged to be at low risk of bias for all domains for this result.

- 'Some concerns': the study is judged to raise some concerns in at least one domain for this result, but not to be at high risk of bias for any domain.

- 'High risk of bias': the study is judged to be at high risk of bias in at least one domain for the result or the study is judged to have some concerns for multiple domains in a way that substantially lowers confidence in the results.

We will use the RoB 2 Excel tool to implement RoB 2 (available on the riskofbiasinfo.org website) and will store and present our detailed RoB 2 assessments as supplementary online material. We will use the Risk-of-bias VISualization tool (robvis) to generate risk of bias summary figures (McGuinness 2020).

\section{Measures of treatment effect}

For continuous outcomes, we will record the mean, standard deviation and total number of participants in both the treatment and control groups. For dichotomous outcomes, we will record the number of events and total number of participants in both the treatment and control groups.

For continuous outcomes measured using the same scale, we will perform analyses using the mean difference (MD) with $95 \%$ confidence intervals (Cls). For continuous outcomes measured with different scales we will perform analyses using the standardised mean difference (SMD). For interpreting SMDs, we will re-express SMDs in the original units of a particular scale with the most clinical relevance and impact (e.g. clinical symptoms with the WHO Clinical Progression Scale (WHO 2020f)).

If available, we will extract and report hazard ratios (HRs) for timeto-event outcomes (time to death). If HRs are not available, we will make every effort to estimate the HR as accurately as possible using the available data and a purpose-built method based on the Parmar and Tierney approach (Parmar 1998; Tierney 2007). If sufficient studies provide HRs, we will use HRs rather than risk ratios (RRs) or MDs in a meta-analysis.

For dichotomous outcomes, we will report the pooled risk ratio (RR) with a $95 \% \mathrm{Cl}$ (Deeks 2020). If the number of observed events is small (less than $5 \%$ of sample per group), and if studies have balanced treatment groups, we will report the Peto odds ratio (OR) with $95 \% \mathrm{Cl}$ (Deeks 2020).

\section{Unit of analysis issues}

\section{Studies with multiple treatment groups}

As recommended in Chapter 6 of the Cochrane Handbook for Systematic Reviews of Interventions (Higgins 2020a), for studies with multiple treatment groups, we will combine arms as long as they can be regarded as subtypes of the same intervention. When arms can not be pooled this way, we will compare each arm with the 
common comparator separately. For pair-wise meta-analysis, we will split the 'shared' group into two or more groups with smaller sample sizes, and include two or more (reasonably independent) comparisons. For this purpose, for dichotomous outcomes, both the number of events and the total number of participants will be divided up, and for continuous outcomes, the total number of participants will be divided up with unchanged means and SDs.

\section{Dealing with missing data}

Chapter 6 of the Cochrane Handbook for Systematic Reviews of Interventions suggests a number of potential sources for missing data, which we will take into account: at study level, at outcome level and at summary data level (Higgins 2020a). In the first instance, it is of the utmost importance to differentiate between data 'missing at random' and 'not missing at random'.

If data are missing, we will request these data from the principal investigators. If, after this, data are still missing, we will have to make explicit assumptions of any methods the included studies used. For example, we will assume that the data were missing at random or we will assume that missing values had a particular value, such as a poor outcome.

\section{Assessment of heterogeneity}

We will assess heterogeneity of treatment effects between studies using a $\mathrm{Chi}^{2}$ test with a significance level at $\mathrm{P}<0.1$. We will use the $I^{2}$ statistic (Higgins 2003), to quantify possible heterogeneity $\left(\mathrm{I}^{2}\right.$ statistic $>30 \%$ to signify moderate heterogeneity, $\mathrm{I}^{2}$ statistic $>75 \%$ to signify considerable heterogeneity; Deeks 2020). If heterogeneity is above $80 \%$, we will explore potential causes through sensitivity and subgroup analyses. If we cannot find a reason for heterogeneity, we will not perform a meta-analysis, but will comment on results from all studies and present these in tables.

\section{Assessment of reporting biases}

As mentioned above, we will search trials registries to identify completed studies that have not been published elsewhere, to minimise or determine publication bias. We intend to explore potential publication bias by generating a funnel plot and statistically testing this by conducting a linear regression test for meta-analyses involving at least 10 studies (Sterne 2019). We will consider $\mathrm{P}<0.1$ as significant for this test.

\section{Data synthesis}

If the clinical and methodological characteristics of individual studies is sufficiently homogeneous, we will pool the data in meta-analysis. We will perform analyses according to the recommendations of the Cochrane Handbook for Systematic Reviews of Interventions (Deeks 2020). We will conduct separate meta-analyses for each comparison.

We will use the Review Manager Web software for analyses (RevMan Web 2019). One review author will enter the data into the software, and a second review author will check the data for accuracy.

We will include all eligible studies in meta-analysis and will assess the effects of potential biases in sensitivity analyses (see Sensitivity analysis).

When meta-analysis is feasible, we will use the random-effects model for pooling the data as we anticipate that true effects will be related but will not be the same for included studies. For binary outcomes, we will base the estimation of the between-study variance using the Mantel-Haenszel method. We will use the inverse variance method for continuous outcomes, outcomes that include data from cluster-RCTs, or outcomes where HRs are available. We will explore heterogeneity above $80 \%$ with subgroup analyses. If we cannot find a cause for the heterogeneity or data are not reported homogeneously, then we will not perform a meta-analysis, but comment on the results as a narrative synthesis with the results from all studies presented in tables.

\section{Subgroup analysis and investigation of heterogeneity}

We will perform subgroup analyses of the following characteristics.

- Age of participants (divided into applicable age groups, e.g. children; 18 to 65 years, 65 years and older)

- Pre-existing conditions (diabetes, respiratory disease, hypertension, immunosuppression)

- Occupation (e.g. health worker, grocery retail, cabin crew)

- Mode of exposure (e.g. workplace, nursing home)

- Burden of exposure; this item will address how much/intensive included participants are exposed to COVID-19 (e.g. in regular contact with infected people through occupation, living in high-risk area; not directly exposed but high-risk medical background).

We will use the tests for interaction to test for differences between subgroup results.

\section{Sensitivity analysis}

We will perform sensitivity analysis of the following characteristics for our primary outcomes:

- 'Risk of bias' assessment components (studies with a low risk of bias or some concerns versus studies with a high risk of bias)

- Influence of preprints of COVID-19 interventions versus peerreviewed articles

- Influence of premature termination of studies

\section{Summary of findings and assessment of the certainty of the evidence}

\section{Assessment of the certainty of the evidence}

We will use the GRADE approach to assess the certainty of the evidence for the following outcomes. The GRADE approach uses five considerations (risk of bias, consistency of effect, imprecision, indirectness and publication bias) to assess the certainty in the body of evidence for each prioritised outcome, and to draw conclusions about the certainty of evidence within the text of the review. We will follow the current GRADE guidance for these assessments in its entirety as recommended in the Cochrane Handbook for Systematic Reviews of Interventions, Chapter 14 (Schünemann 2020).

We will use the overall 'Risk of bias' judgement, derived from the RoB 2 Excel tool, to inform our decision on downgrading for risk of bias. For time-to-event outcomes we will calculate absolute effects at specific time points as recommended in the GRADE guidance 27 (Skoetz 2020). We will phrase the findings and certainty of the evidence as suggested in the informative statement guidance (Santesso 2020). 


\section{'Summary of findings' table}

We will use GRADEpro GDT software (GRADEpro GDT) to create a 'Summary of findings' table, as suggested in the Cochrane Handbook for Systematic Reviews of Interventions (Schünemann 2020).

According to Chapter 14 of the updated Cochrane Handbook for Systematic Reviews of Interventions, the "most critical and/ or important health outcomes, both desirable and undesirable" should be included in the 'Summary of findings' table(s) (Schünemann 2019). As previously described, we will include outcomes prioritised according to the Core Outcome Set for studies evaluating public health, primary and secondary care interventions for prevention of COVID-19 transmission (COMET 2020). Those are:

- infection with SARS-CoV-2 (positive RT-PCR test) within 30 days

- development of clinical COVID-19 symptoms, assessed with the WHO Clinical Progression Scale (WHO 2020f) within 30 days

- all-cause mortality until longest follow-up

- mortality (time to event)

- admission to hospital within 30 days

- quality of life, assessed with standardised scales (e.g. WHOQOL-100) at up to seven days; up to 30 days, and longest follow-up available

- adverse events, including if possible imputability of events

- serious adverse events, including if possible imputability of events

\section{Living systematic review considerations}

Following the guidance for the production and publication of Cochrane living systematic reviews (Cochrane LSR 2019), whenever new evidence (meaning studies, data or other information) relevant to the review is identified, we will extract the data and assess risk of bias, as appropriate. We will wait until the accumulating evidence changes one or more of the following components of the review before incorporating it and republishing the review:

- the findings of one or more prioritised outcomes;
- the credibility (e.g. GRADE rating) of one or more prioritised outcomes;

- new settings, populations, interventions, comparisons or outcomes studied.

We will review the review scope and methods approximately monthly, or more frequently if appropriate in light of potential changes in COVID-19 research (for example, when additional comparisons, interventions, subgroups or outcomes, or new review methods become available).

\section{ACKNOWLEDGEMENTS}

This review was published in collaboration with the Cochrane Editorial and Methods Department. We particularly thank Clare Dooley (Managing Editor) for her excellent support. Thanks also to the Cochrane Editorial and Methods Department team, for their valuable comments on the review protocol and timely management of the editorial process.

We thank all referees who read and commented on this protocol. We thank Robin Featherstone (Information Specialist, Cochrane Editorial and Methods Department) for commenting on the search strategy. We thank Chantelle Garritty (Methodology Editor, Editorial and Methods Department) and Prof DM Altmann (topic expert reviewer) for reviewing and commenting on our planned methodology and the public health relevance. Thanks to Denise Mitchell for copy-editing the protocol.

The research was supported by NHS Blood and Transplant and the National Institute for Health Research (NIHR) Oxford Biomedical Research Centre (BRC). The views expressed are those of the author(s) and not necessarily those of the NHS, the NIHR or the Department of Health.

The contents of this document reflects only the authors' view and the Commission is not responsible for any use that may be made of the information it contains. This project has received funding from the European Union's Horizon 2020 research and innovation programme under grant agreement No. 101015756. 


\section{R E F E R E N C E S}

\section{Additional references}

\section{Bao 2020}

Bao L, Deng W, Gao H, Xiao C, Liu J, Xue J, et al. Reinfection could not occur in SARS-CoV-2 infected rhesus macaques. bioRxiv [Preprint] 2020. [DOI: 10.1101/2020.03.13.990226]

\section{Baudel 2020}

Baudel JL, Vigneron C, Pras-Landre V, Joffre J, Marjot F, AitOufella $\mathrm{H}$, et al. Transfusion-related acute lung injury (TRALI) after intravenous immunoglobulins: French multicentre study and literature review. Clinical Rheumatology 2020;2:541-6.

\section{Beigel 2019}

Beigel JH, Aga E, Elie-Turenne MC, Cho J, Tebas P, Clark CL, et al. Anti-influenza immune plasma for the treatment of patients with severe influenza A: a randomised, double-blind, phase 3 trial. Lancet Respiratory Medicine 2019;7(11):941-50.

\section{Beigel 2020}

Beigel JH, Tomashek KM, Dodd LE, Mehta AK, Zingman BS, Kalil AC, et al. Remdesivir for the treatment of COVID-19 - preliminary report. New England Journal of Medicine 2020;383(10):994. [DOI: 10.1056/NEJMoa2007764]

\section{Bloch 2020}

Bloch EM, Shoham S, Casadevall A, Sachais BS, Shaz B, Winters JL, et al. Deployment of convalescent plasma for the prevention and treatment of COVID-19. Journal of Clinical Investigation 2020;130(6):2757-65.

\section{Boulware 2020}

Boulware DR, Pullen MF, Bangdiwala AS, Pastick KA, Lofgren SM, Okafor EC, et al. A randomized trial of hydroxychloroquine as postexposure prophylaxis for COVID-19. New England Journal of Medicine 2020;383(6):517-25.

\section{Brennan 2003}

Brennan VM, Salomé-Bentley NJ, Chapel HM, Immunology Nurses Study. Prospective audit of adverse reactions occurring in 459 primary antibody-deficient patients receiving intravenous immunoglobulin. Clinical and Experimental Immunology 2003;133(2):247-51.

\section{Canelo-Aybar 2020}

Canelo-Aybar C, Beltran J, Santero M, Alonso-Coello P. Adjusted fatality rates of COVID19 pandemic: a comparison across countries. medRxiv [Preprint] 2020. [DOI: 10.1101/2020.05.13.20099796]

\section{Casadevall 2020}

Casadevall A, Pirofski LA. The convalescent sera option for containing COVID-19. Journal of Clinical Investigation 2020;130(4):1545-8.

\section{CDC 2020a}

Centers for Disease Control and Prevention (CDC). Rabies postexposure prophylaxis (PEP). www.cdc.gov/rabies/ medical_care/index.html (accessed 24 August 2020).

\section{CDC 2020b}

Centers for Disease Control and Prevention (CDC). Postexposure prophylaxis. www.cdc.gov/hepatitis/hbv/pep.htm (accessed 24 August 2020).

\section{CDC 2020C}

Centers for Disease Control and Prevention (CDC). Interim clinical guidance for management of patients with confirmed coronavirus disease (COVID-19). www.cdc.gov/ coronavirus/2019-ncov/hcp/clinical-guidance-managementpatients.html (accessed 24 August 2020).

\section{Chai 2020}

Chai KL, Valk SJ, Piechotta V, Kimber C, Monsef I, Wood EM, et al. Convalescent plasma or hyperimmune immunoglobulin for people with COVID-19: a living systematic review. Cochrane Database of Systematic Reviews 2020, Issue 7. Art. No: CD013600. [DOI: 10.1002/14651858.CD013600.pub3]

\section{Chen L 2020}

Chen L, Xiong J, Bao L, Shi Y. Convalescent plasma as a potential therapy for COVID-19. Lancet Infectious Diseases 2020;20(4):398400.

\section{Chen Z 2020}

Chen Z, Wherry EJ. T cell responses in patients with COVID-19. Nature Reviews. Immunology 2020;20(9):529-36. [DOI: 10.1038/ s41577-020-0402-6]

\section{Chun 2016}

Chun S, Chung CR, Ha YE, Han TH, Ki CS, Kang ES, et al. Possible transfusion-related acute lung injury following convalescent plasma transfusion in a patient with Middle East respiratory syndrome. Annals of Laboratory Medicine 2016;36(4):393-5.

\section{Cochrane LSR 2019}

Guidance for the production and publication of Cochrane living systematic reviews: Cochrane Reviews in living mode. community.cochrane.org/review-production/productionresources/living-systematic-reviews (accessed 4 August 2020).

\section{COMET 2020}

A Core Outcome Set for studies evaluating public health, primary and secondary care interventions for prevention of COVID-19 transmission - the COS-COVID-P study. www.cometinitiative.org/Studies/Details/1594 (accessed 3 August 2020).

\section{Covidence [Computer program]}

Veritas Health Innovation Covidence. Melbourne, Australia: Veritas Health Innovation. Available at covidence.org.

\section{Deeks 2020}

Deeks JJ, Higgins JP, Altman DG, editor(s). Chapter 10: Analysing data and undertaking meta-analyses. In: Higgins JP, Thomas J, Chandler J, Cumpston M, Li T, Page MJ, et al, editor(s). Cochrane Handbook for Systematic Reviews of Interventions version 6.1 (updated September 2020). Cochrane, 2020. Available from training.cochrane.org/handbook. 


\section{Driggin 2020}

Driggin E, Madhavan MV, Bikdeli B, Chuich T, Laracy J, BiondiZoccai G, et al. Cardiovascular considerations for patients, health care workers, and health systems during the COVID-19 pandemic. Journal of the American College of Cardiology 2020;75(18):2352-71.

\section{D'Antiga 2020}

D'Antiga L. Coronaviruses and immunosuppressed patients: the facts during the third epidemic. Liver Transplantation 2020;26(6):832-4.

\section{Eibl 2008}

Eibl MM. History of immunoglobulin replacement. Immunology and Allergy Clinics of North America 2008;28(4):737-64.

\section{Eldridge 2016}

Eldridge S, Campbell M, Campbell M, Dahota A, Giraudeau B, Higgins JT, et al. Revised Cochrane risk of bias tool for randomized trials (RoB 2.0) Additional considerations for cluster-randomized trials. www.riskofbias.info/welcome/ rob-2-0-tool/archive-rob-2-0-cluster-randomized-trials-2016 (accessed 4 August 2020).

\section{Fung 2020}

Fung M, Babik JM. COVID-19 in immunocompromised hosts: what we know so far. Clinical Infectious Diseases 2020 Jun 27 [Epub ahead of print]. [DOI: 10.1093/cid/ciaa863]

\section{Garg 2020}

Garg S, Kim L, Whitaker M, O'Halloran A, Cummings C, Holstein $\mathrm{R}$, et al. Hospitalization rates and characteristics of patients hospitalized with laboratory-confirmed coronavirus disease 2019 - COVID-NET, 14 States, March 1-30, 2020. Morbidity and Mortality Weekly Report 2020;69:458-64. [DOI: 10.15585/mmwr.mm6915e3]

\section{GRADEpro GDT [Computer program]}

McMaster University (developed by Evidence Prime) GRADEpro GDT. Hamilton (ON): McMaster University (developed by Evidence Prime). Available at gradepro.org.

\section{Higgins 2003}

Higgins JP, Thompson SG, Deeks JJ, Altman DG. Measuring inconsistency in meta-analyses. BMJ 2003;327:557-60.

\section{Higgins 2020a}

Higgins JP, Tianging L, Deeks JJ, editor(s). Chapter 6: Choosing effect measures and computing estimates of effect. In: Higgins JP, Thomas J, Chandler J, Cumpston M, Li T, Page MJ, et al, editor(s). Cochrane Handbook for Systematic Reviews of Interventions Version 6.1 (updated September 2020). Cochrane, 2020. Available from training.cochrane.org/handbook.

\section{Higgins 2020b}

Higgins JP, Eldridge S, Li T. Chapter 23: Including variants on randomized trials. In: Higgins JP, Thomas J, Chandler J, Cumpston M, Li T, Page MJ, et al, editor(s). Cochrane Handbook for Systematic Reviews of Interventions Version 6.1 (updated September 2020). Cochrane, 2020. Available from training.cochrane.org/handbook.

\section{Higgins 2020c}

Higgins JP, Savović J, Page MJ, Elbers RG, Sterne JA. Chapter 8: Assessing risk of bias in a randomized trial. In: Higgins JP, Thomas J, Chandler J, Cumpston M, Li T, Page MJ, et al, editor(s). Cochrane Handbook for Systematic Reviews of Interventions Version 6.1 (updated September 2020). Cochrane, 2020. Available from training.cochrane.org/handbook.

\section{Horby 2020}

Horby P, Lim WS, Emberson J, Mafham M, Bell J, Linsell L, et al. Effect of dexamethasone in hospitalized patients with COVID-19: preliminary report. medRxiv [Preprint] 2020. [DOI: 10.1101/2020.06.22.20137273]

\section{Huang 2020}

Huang C, Wang Y, Li X, Ren L, Zhao J, Hu Y, et al. Clinical features of patients infected with 2019 novel coronavirus in Wuhan, China. Lancet 2020;395(10223):497-506.

\section{Hung 2013}

Hung IF, To KK, Lee CK, Lee KL, Yan WW, Chan K, et al. Hyperimmune IV immunoglobulin treatment: a multicenter double-blind randomized controlled trial for patients with severe 2009 influenza $A(H 1 N 1)$ infection. Chest 2013;144(2):464-73.

\section{Ibarrondo 2020}

Ibarrondo FJ, Fulcher JA, Goodman-Meza D, Elliott J, Hofmann C, Hausner MA, et al. Rapid decay of anti-SARS-CoV-2 antibodies in persons with mild COVID-19. New England Journal of Medicine 2020;383(11):e74.

\section{Iwasaki 2020}

Iwasaki A. What reinfections mean for COVID-19. Lancet Infectious Diseases 2020 Oct 12 [Epub ahead of print]. [DOI: 10.1016/S1473-3099(20)30783-0]

\section{Kim 2020}

Kim DH, Choe YJ, Jeong JY. Understanding and interpretation of case fatality rate of coronavirus disease 2019. Journal of Korean Medical Science 2020;35(12):e137.

\section{Lauer 2020}

Lauer SA, Grantz KH, Bi Q, Jones FK, Zheng Q, Meredith HR, et al. The incubation period of coronavirus disease 2019 (COVID-19) from publicly reported confirmed cases: estimation and application. Annals of Internal Medicine 2020;172(9):577-82. [DOI: 10.7326/M20-0504]

\section{Lefebvre 2020}

Lefebvre C, Glanville J, Briscoe S, Littlewood A, Marshall C, Metzendorf M-I, et al. Chapter 4: Searching for and selecting studies. In: Higgins JP, Thomas J, Chandler J, Cumpston M, Li T, Page MJ, et al, editor(s). Cochrane Handbook for Systematic Reviews of Interventions Version 6.1 (updated September 2020). Cochrane, 2020. Available from training.cochrane.org/ handbook.

\section{Li 2020}

Li T, Higgins JP, Deeks JJ. Chapter 5: Collecting data. In: Higgins JP, Thomas J, Chandler J, Cumpston M, Li T, Page MJ, et al, 
editor(s). Cochrane Handbook for Systematic Reviews of Interventions Version 6.1 (updated September 2020). Cochrane, 2020. Available from training.cochrane.org/handbook.

\section{Liang 2020}

Liang W, Guan W, Chen R, Wang W, Li J, Xu K, et al. Cancer patients in SARS-CoV-2 infection: a nationwide analysis in China. Lancet Oncology 2020;21(3):335-7.

\section{Luke 2006}

Luke TC, Kilbane EM, Jackson JL, Hoffman SL. Meta-analysis: convalescent blood products for Spanish influenza pneumonia: a future H5N1 treatment? Annals of Internal Medicine 2006;145(8):599-609.

\section{Mair-Jenkins 2015}

Mair-Jenkins J, Saavedra-Campos M, Baillie JK, Cleary P, Khaw FM, Lim WS, et al. The effectiveness of convalescent plasma and hyperimmune immunoglobulin for the treatment of severe acute respiratory infections of viral etiology: a systematic review and exploratory meta-analysis. Journal of Infectious Diseases 2015;211(1):80-90.

\section{McGuinness 2020}

McGuinness LA, Higgins JP. Risk-of-bias VISualization (robvis): An R package and Shiny web app for visualizing risk-of-bias assessments. Research Synthesis Methods: Special Issue Paper 2020. [DOI: 10.1002/jrsm.1411]

\section{Microsoft Corporation 2018 [Computer program]}

Microsoft Excel. Microsoft Corporation. Microsoft Corporation, 2018. office.microsoft.com/excel.

\section{Mo 2006}

Mo H, Zeng G, Ren X, Li H, Ke C, Tan Y, et al. Longitudinal profile of antibodies against SARS-coronavirus in SARS patients and their clinical significance. Respirology 2006;11(1):49-53. [DOI: 10.1111/j.1440-1843.2006.00783.x]

\section{Moher 2009}

Moher D, Liberati A, Tetzlaff J, Altman DG. Preferred reporting items for systematic reviews and meta-analyses: the PRISMA statement. Journal of Clinical Epidemiology 2009;62(10):1006-12.

\section{Morens 1994}

Morens DM. Antibody-dependent enhancement of infection and the pathogenesis of viral disease. Clinical Infectious Diseases 1994;19(3):500-12.

\section{Ogilvie 1998}

Ogilvie MM. Antiviral prophylaxis and treatment in chickenpox: a review prepared for the UK Advisory Group on Chickenpox on behalf of the British Society for the Study of Infection. Journal of Infection 1998;36:31-8.

\section{Otrock 2017}

Otrock ZK, Liu C, Grossman BJ. Transfusion-related acute lung injury risk mitigation: an update. Vox Sanguinis 2017;112(8):694-703.

\section{Pandey 2012}

Pandey S, Vyas GN. Adverse effects of plasma transfusion. Transfusion 2012;52 Suppl 1:65S-79S.

\section{Parmar 1998}

Parmar MK, Torri V, Stewart L. Extracting summary statistics to perform meta-analyses of the published literature for survival endpoints. Statistics in Medicine 1998;17(24):2815-34.

\section{Payne 2016}

Payne DC, Iblan I, Rha B, Algasrawi S, Hin A, Al Nsour M, et al. Persistence of antibodies against Middle East respiratory syndrome coronavirus. Emerging Infectious Disease Journal 2016;22(10):1824-6. [DOI: 10.3201/eid2210.160706]

\section{PREVENT 1997}

PREVENT Study Group. Reduction of respiratory syncytial virus hospitalization among premature infants and infants with bronchopulmonary dysplasia using respiratory syncytial virus immune globulin prophylaxis. Pediatrics 1997;99(1):93-9.

\section{RevMan Web 2019 [Computer program]}

The Cochrane Collaboration Review Manager Web (RevMan Web). The Cochrane Collaboration, 2019. revman.cochrane.org.

\section{Robbins 1995}

Robbins JB, Schneerson R, Szu SC. Perspective: hypothesis: serum IgG antibody is sufficient to confer protection against infectious diseases by inactivating the inoculum. Journal of Infectious Diseases 1995;171(6):1387-98.

\section{Rock 2011}

Rock G. A comparison of methods of pathogen inactivation of FFP. Vox Sanguinis 2011;100(2):169-78.

\section{Russell 2020}

Russell TW, Hellewell J, Jarvis Cl, Van ZK, Abbott S, Ratnayake R, et al. Estimating the infection and case fatality ratio for coronavirus disease (COVID-19) using age-adjusted data from the outbreak on the Diamond Princess cruise ship, February 2020. Euro Surveillance 2020;25(12):2000256.

\section{Santesso 2020}

Santesso N, Glenton C, Dahm P, Garner P, Akl A, Alper B, et al. GRADE guidelines 26: informative statements to communicate the findings of systematic reviews of interventions. Journal of Clinical Epidemiology 2020;119:126-35.

\section{Schünemann 2020}

Schünemann HJ, Vist GE, Higgins JP, Santesso N, Deeks JJ, Glasziou P, et al. Chapter 15: Interpreting results and drawing conclusions. In: Higgins JP, Thomas J, Chandler J, Cumpston M, Li T, Page MJ, et al, editor(s). Cochrane Handbook for Systematic Reviews of Interventions Version 6.1 (updated September 2020). Cochrane, 2020. Available from training.cochrane.org/ handbook.

\section{Sekul 1994}

Sekul EA, Cupler EJ, Dalakas MC. Aseptic meningitis associated with high-dose intravenous immunoglobulin therapy: 
frequency and risk factors. Annals of Internal Medicine 1994;121(4):259-62.

\section{Shen 2020}

Shen C, Wang Z, Zhao F, Yang Y, Li J, Yuan J, et al. Treatment of 5 critically ill patients with COVID-19 with convalescent plasma. JAMA 2020;323(16):1582-9.

\section{Skoetz 2020}

Skoetz N, Goldkuhle M, Van Dalen EC, Akl EA, Trivella M, Mustafa RA, et al. GRADE guidelines 27: how to calculate absolute effects for time-to-event outcomes in summary of findings tables and evidence profiles. Journal of Clinical Epidemiology 2020;118:124-31.

\section{Soto 2020}

Soto JA, Gálvez NM, Pacheco GA, Bueno SM, Kalergis AM. Antibody development for preventing the human respiratory syncytial virus pathology. Molecular Medicine 2020;26(1):35.

\section{Sterne 2019}

Sterne JA, Savovic J, Page MJ, Elbers RG, Blencowe N, Boutron I, et al. RoB 2: a revised tool for assessing risk of bias in randomised trials. BMJ 2019;366:14898.

\section{Stiehm 2013}

Stiehm ER. Adverse effects of human immunoglobulin therapy. Transfusion Medicine Reviews 2013;27(3):171-8.

\section{Team NCPERE 2020}

Team NCPERE . Vital surveillances: the epidemiological characteristics of an outbreak of 2019 novel coronavirus diseases (COVID-19) - China. China CDC Weekly 2020;2(8):113-22.

\section{Tierney 2007}

Tierney JF, Stewart LA, Ghersi D, Burdett S, Sydes MR. Practical methods for incorporating summary time-to-event data into meta-analysis. Trials 2007;8:16.

\section{Tolouian 2020}

Tolouian R, Vahed SZ, Ghiyasvand S, Tolouian A, Ardalan M. COVID-19 interactions with angiotensin-converting enzyme 2 (ACE2) and the kinin system; looking at a potential treatment. Journal of Renal Injury Prevention 2020;9(2):e19.

\section{US Covid Plasma 2020}

US Covid Plasma. COVID-19 expanded access program. www.uscovidplasma.org (accessed 9 July 2020).

\section{Van de Veerdonk 2020}

Van de Veerdonk F, Netea MG, Van Deuren M, Van der Meer JW, De Mast Q, Bruggemann R, et al. Kinins and cytokines in COVID-19: a comprehensive pathophysiological approach. Preprints [Preprint] 2020. [DOI: 10.20944/ preprints202004.0023.v1]

\section{Wan 2020}

Wan Y, Shang J, Sun S, Tai W, Chen J, Geng Q, et al. Molecular mechanism for antibody-dependent enhancement of coronavirus entry. Journal of Virology 2020;94(5):e02015-19.

\section{Wang 2014}

Wang S-F, Tseng S-P, Yen C-H, Yang J-Y, Tsao C-H, Shen C-W, et al. Antibody-dependent SARS coronavirus infection is mediated by antibodies against spike proteins. Biochemical and Biophysical Research Communications 2014;451(2):208-14.

\section{WHO 2007}

World Health Organization (WHO). Cumulative number of reported probable cases of SARS. www.who.int/csr/sars/ country/2003_07_11/en/ (accessed 13 April 2020).

\section{WHO 2019}

World Health Organization (WHO). Middle East respiratory syndrome coronavirus (MERS-CoV). www.who.int/emergencies/ mers-cov/en/ (accessed 13 April 2020).

\section{WHO 2020a}

World Health Organization (WHO). Report of the WHOChina Joint Mission on coronavirus disease 2019 (COVID-19). www.who.int/docs/default-source/coronaviruse/who-chinajoint-mission-on-covid-19-final-report 2020.

\section{WHO 2020b}

World Health Organization (WHO). WHO Coronavirus Disease (COVID-19) Dashboard. covid19.who.int (accessed 23 November 2020).

\section{WHO 2020c}

World Health Organization (WHO). Weekly epidemiological update - 17 November 2020. www.who.int/publications/m/ item/weekly-epidemiological-update-17-november-2020 2020.

\section{WHO 2020d}

World Health Organization (WHO). Estimating mortality from COVID-19. www.who.int/news-room/commentaries/detail/ estimating-mortality-from-covid-19 2020.

\section{WHO 2020e}

World Health Organization (WHO). Clinical management of COVID-19 - interim guidance, 27 May 2020. WHO/2019-nCoV/ clinical/2020.4.

\section{WHO $2020 f$}

WHO working group on the clinical characterisation and management of COVID-19 infection. A minimal common outcome measure set for COVID-19 clinical research. Lancet Infectious Diseases 2020;20(8):e192-7.

\section{Williamson 2020}

Williamson E, Walker AJ, Bhaskaran KJ, Bacon S, Bates C, Morton CE, et al, OpenSAFELY Collaborative. OpenSAFELY: factors associated with COVID-19-related hospital death in the linked electronic health records of 17 million adult NHS patients. medRxiv [Preprint] 2020. [DOI: 10.1101/2020.05.06.20092999]

\section{Wu 2020a}

Wu F, Wang A, Liu M, Wang Q, Chen J, Xia S, et al. Neutralizing antibody responses to SARS-CoV-2 in a COVID-19 recovered patient cohort and their implications. medRxiv [Preprint] 2020. [DOI: $10.1101 / 2020.03 .30 .20047365]$ 


\section{Wu 2020b}

Wu F, Zhao S, Yu B, Chen Y-M, Wang W, Song ZG, et al. A new coronavirus associated with human respiratory disease in China. Nature 2020;579(7798):265-9. [DOI: 10.1038/ s41586-020-2008-3]

\section{Xu 2020}

Xu Z, Shi L, Wang Y, Zhang J, Huang L, Zhang C, et al. Pathological findings of COVID-19 associated with acute respiratory distress syndrome. Lancet Respiratory Medicine 2020;8(4):420-2.

\section{AP P E N D I C E S}

\section{Appendix 1. Search strategy MEDLINE}

1. Coronavirus Infections/

2. Coronavirus/

3. "Betacoronavirus"/

4. ((corona* or corono*) adj1 (virus* or viral ${ }^{\star}$ or virinae $\left.\left.{ }^{\star}\right)\right)$. tw,kf.

5. (coronavirus* or coronovirus $^{\star}$ or coron?virinae* or "2019-nCoV" or 2019nCoV or 2019-CoV or nCoV2019 or "nCoV-2019" or "COVID-19" or COVID19 or "CORVID-19" or CORVID19 or "WN-CoV" or WNCoV or "HCoV-19" or HCoV19 or CoV or "2019 novel*" or Ncov or "n-cov" or "SARSCoV-2" or "SARSCoV-2" or "SARSCoV2" or "SARS-CoV2" or SARSCov19 or "SARS-Cov19" or "SARSCov-19" or "SARS-Cov-19" or SARSr-Cov or Ncovor or Ncorona* or Ncorono* or NcovWuhan* or NcovHubei* or NcovChina* or NcovChinese* or Wuhan virus* or novel CoV or CoV 2 or CoV2 or betacoron?vir*).tw,kf.

6. (((respiratory ${ }^{\star}$ adj2 (acute* or symptom* or disease* or illness ${ }^{\star}$ or condition*)) or "sea-food market*" or "seafood market" or "food market*" or "foodmarket*") adj10 (Wuhan* or Hubei* or China* or Chinese* or Huanan*)).tw,kf.

7. ((outbreak* or wildlife* or wild-life or pandemic* or epidemic ${ }^{\star}$ ) adj3 (Wuhan* or Hubei* or China* or Chinese* or Huanan*)).tw,kf.

8. (anti-flu* or anti-influenza* or antiflu* or antinfluenza*).tw,kf.

9. 1 or 2 or 3 or 4 or 5 or 6 or 7 or 8

10. Plasma/

11. Immunoglobulins/

12. Immunoglobulins, Intravenous/

13. Immune Sera/

14. ((convalesc ${ }^{\star}$ or recovered or cured or rehabilitat* or survivor $^{\star}$ or survived or virus-positive or virus neutrali* or virus inactivated or antibod $^{\star}$ or high-titre* or high-titer ${ }^{\star}$ ) adj6 (plasma or blood or serum or sera)).mp.

15. ((plasma adj1 therap*) or gamma-globulin* or " $\gamma$-Globulin" or hyper-Ig).tw,kf.

16. ((hyperimmune or hyper-immune or high-dos*) adj3 (plasma or immunoglobulin* or IVIG* or immune globulin* or globulin* or $\lg G)) . t w, k f$.

17. (plasma adj5 (immun* or antibod* or exchange $^{\star}$ or donor $^{\star}$ or donat ${ }^{\star}$ or transfus ${ }^{\star}$ or infus $\left.{ }^{\star}\right)$ ).mp.

18. ((convalesc ${ }^{\star}$ or recovered or cured or rehabilitat* or survivor ${ }^{\star}$ or survived or virus-positive or virus inactivated or antibody-positive) $\operatorname{adj5}\left(\right.$ donor $^{\star}$ or donat*)).mp.

19. (((serum or sera) adj2 (therap* or treatment $\left.\left.^{\star}\right)\right)$ or serotherap ${ }^{\star}$ or sero-therap $\left.{ }^{\star}\right)$. tw,kf.

20.10 or 11 or 12 or 13 or 14 or 15 or 16 or 17 or 18 or 19

21.9 and 20

Convalescent plasma and hyperimmune immunoglobulin to prevent infection with SARS-CoV-2 (Protocol) Copyright @ 2020 The Cochrane Collaboration. Published by John Wiley \& Sons, Ltd. 
22. Covid-19 Serotherapy.px

23. (Flu-IVIG or ((anti-flu* or anti-influenza* or antiflu* or antinfluenza*) adj5 plasma)).mp.

24. 21 or 22 or 23

25. (exp Animals/ or exp Animal Experimentation/ or exp Models, Animal/) not Humans/

26. 24 not 25

27. limit 26 to $y r=" 2019$-Current"

\section{Appendix 2. Search strategy Embase}

1. "Coronavirus Infections"/ or "Coronavirus Infection"/

2. Coronavirinae/ or Coronavirus/ or exp Betacoronavirus/

3. ((corona* or corono $\left.{ }^{\star}\right)$ adj1 (virus ${ }^{\star}$ or viral* or virinae $\left.\left.{ }^{\star}\right)\right) . \mathrm{tw}, \mathrm{kw}$.

4. (coronavirus* or coronovirus* or coron?virinae* or "2019-nCoV" or 2019nCoV or 2019-CoV or nCoV2019 or "nCoV-2019" or "COVID-19" or COVID19 or "CORVID-19" or CORVID19 or "WN-CoV" or WNCoV or "HCoV-19" or HCoV19 or CoV or "2019 novel " or Ncov or "n-cov" or "SARSCoV-2" or "SARSCoV-2" or "SARSCoV2" or "SARS-CoV2" or SARSCov19 or "SARS-Cov19" or "SARSCov-19" or "SARS-Cov-19" or SARSr-Cov or Ncovor or Ncorona* or Ncorono* or NcovWuhan" or NcovHubei* or NcovChina* or NcovChinese* or Wuhan virus* or novel CoV or CoV 2 or $\mathrm{CoV} 2$ or betacoron?vir*).tw,kw.

5. (( (respiratory ${ }^{\star}$ adj2 (acute* or symptom* or disease* or illness ${ }^{\star}$ or condition*)) or "sea-food market*" or "seafood market ${ }^{\star} "$ or "food

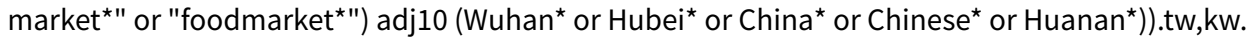

6. ((outbreak* or wildlife* or wild-life* or pandemic ${ }^{\star}$ or epidemic ${ }^{\star}$ ) adj3 (Wuhan* or Hubei ${ }^{\star}$ or China* or Chinese* or Huanan*)).tw,kw.

7. (anti-flu* or anti-influenza* or antiflu* or antifluenza*).tw,kw.

8. or $11-7$

9. Plasma Transfusion/

10. exp Immunoglobulin/

11. ((convalesc ${ }^{\star}$ or recovered or cured or survivor ${ }^{\star}$ or survived or rehabilitat ${ }^{\star}$ or virus-positive or virus-neutrali ${ }^{\star}$ or virusinactived or antibody-rich or high-tire* or high-titer ${ }^{\star}$ ) adj6 (plasma or blood or serum or sera)).mp.

12. ((plasma adj1 therap*) or gamma-globulin or "y-Globulin" or hyper-lg).tw,kw.

13. (plasma adj5 (immun* or antibod* or exchange or donor $^{\star}$ or donat ${ }^{\star}$ or transfus ${ }^{\star}$ or infus or $\left.^{\star}\right)$.mp.

14. ((convalesc ${ }^{\star}$ or recovered or cured or survivor ${ }^{\star}$ or rehabilitat ${ }^{\star}$ or survived or virus-positive or virus inactived or antibody-positive) adj5 $\left(\right.$ donor $^{\star}$ or donat $\left.\left.{ }^{\star}\right)\right) \cdot \mathrm{mp}$.

15. (plasma adj5 (immun* or antibod ${ }^{\star}$ or exchange* or donor $^{\star}$ or donat ${ }^{\star}$ or transfus ${ }^{\star}$ or infus $\left.{ }^{\star}\right)$ ).mp.

16. ((hyperimmune or hyper-immune or high-dos*) adj3 (plasma or immunoglobulin* or IVIG* or immune globulin* or globulin* or $\operatorname{lgG})$ ).tw,kw.

17. (plasma adj5 (immun* or antibod* or exchange* or donor $^{\star}$ or donat* or transfus ${ }^{\star}$ or infus (imp. $^{\star}$.mp.

18 ((convalesc ${ }^{\star}$ or recovered or cured or rehabilitat ${ }^{\star}$ or survivor $^{\star}$ or survived or virus-positive or virus inactivated or antibody-positive) $\operatorname{adj5}\left(\right.$ donor $^{\star}$ or donat $\left.\left.^{\star}\right)\right)$.mp.

19. (((serum or sera) adj2 (therap* or treatment $\left.\left.^{\star}\right)\right)$ or serotherap* or sero-therap $\left.{ }^{\star}\right)$.tw,kw.

20. or/9-19

21. (Flu-IVIG or ((anti-flu* or antiflu*) adj5 plasma)).mp.

22. (8 and 20$)$ or 21 
23. (exp animal/ or nonhuman/) not exp human/

24. a nimal experiment/ not (human experiment/ or human/)

25. 23 or 24

26. 22 not 25

\section{Appendix 3. Search strategy PubMed}

\#1 (corona-virus ${ }^{\star}$ OR corono-virus ${ }^{\star}$ OR coronavirus* OR coronovirus ${ }^{\star}$ OR coronavirinae* OR coronovirinae* OR betacoronavirus OR Wuhan* OR Hubei* OR Huanan OR "2019 nCoV" OR 2019nCoV OR 2019 CoV OR nCoV2019 OR "nCoV 2019" OR "COVID 19" OR COVID19 OR "CORVID 19" OR CORVID19 OR "WN CoV" OR WNCoV OR "HCoV 19" OR HCoV19 OR CoV OR "2019 novel" OR Ncov OR "n cov" OR "SARS CoV 2" OR "SARSCOV 2" OR "SARS-CoV-2" OR "SARSCoV-2" OR "SARSCOV2" OR "SARS CoV2" OR „SARS-Cov2“ OR SARSCOV19 OR "SARS Cov19" OR "SARSCov 19" OR "SARS Cov 19" OR Ncovor OR Ncorona* OR Ncorono* OR NcovWuhan* OR NcovHubei* OR NcovChina* OR NcovChinese* OR novel CoV OR CoV2 OR SARSr-cov)v 19" OR "SARS Cov 19" OR Ncovor OR Ncorona* OR Ncorono* OR NcovWuhan* OR NcovHubei* OR NcovChina* OR NcovChinese* OR SARSr-cov)

\#2 (( (respiratory* AND (acute* OR symptom* OR disease OR diseases OR diseased OR illness* OR condition*)) OR "seafood market*" OR "sea food market*" OR "food market*" OR "foodmarket*") AND (Wuhan* OR Hubei* OR China OR "China's" OR Chinese* OR Huanan*))

\#3 ((outbreak* OR wildlife* OR wild-life* OR pandemic* OR epidemic*) AND (China OR "China's" OR Chinese* OR Huanan* OR Wuhan OR Hubei*))

\#4 (anti-flu* OR anti-influenza* OR antiflu* OR antinfluenza*)

\#5 \#1 OR \#2 OR \#3 OR \#4

\#7 ( convalesc ${ }^{\star}[T I A B]$ OR recovered[TIAB] OR cured[TIAB] OR survivor*[TIAB] OR survived[TIAB] OR virus-positive[TIAB] OR virusneutrali* $[T I A B]$ OR "virus inactivated" $[T I A B]$ OR antibod ${ }^{\star}[T I A B]$ OR high-titre* $\left[\right.$ TIAB] OR high-titer ${ }^{\star}$ ) AND (plasma[TIAB] OR blood[TIAB] OR donor $^{\star}[T I A B]$ OR donat $\left.\left.{ }^{\star}[T I A B]\right)\right)$

\#8 ("therapeutic plasma" OR "plasma therapy" OR "immune plasma" OR "plasma exchange" OR gamma-globulin* or "Y-Globulin" or hyper-lg)

\#9 (plasma[TI] AND (immun*[TIAB] OR transfus* [TIAB] OR infus* $[$ TIAB]))

\#10 ((hyperimmune OR hyper-immune OR high-dos ${ }^{\star}$ ) AND (plasma OR immunoglobulin* OR IVIG* OR immune globulin* OR globulin $\left.{ }^{\star}\right)$ )

\#11 \#7 OR \#8 OR \#9 OR \#10

\#12 \#6 AND \#11

\#13 (Flu-IVIG OR ((anti-flu* or anti-influenza* or antiflu* or antinfluenza*) AND plasma))

\#14 \#12 OR \#13

\#15 (publisher[sb] OR inprocess[sb] OR pubmednotmedline[sb])

\#16 \#13 AND \#15: Publication date from 2019/11/01 to present

\section{Appendix 4. Search strategy - World Health Organization COVID-19 Global literature on coronavirus disease}

(tw:(plasma)) OR (tw:(hyperimmune)) OR (tw:(hyper-immune)) OR (tw:(IVIG)) OR (tw:(immunoglobulin*)) OR (tw:(immune-globulin*)) OR (tw:(globulin*)) OR (tw:(gamma-globulin)) OR (tw:(y-Globulin )) OR (tw:(hyper-lg)) OR (tw:(convalesc*)) OR (tw:(sera)) OR (tw:(donor)) OR (tw:(donat $\left.\left.{ }^{\star}\right)\right)$ OR (tw:(sero*)) OR (tw:(flu-IVIG)) OR (tw:(antiflu*)) OR (tw:(anti-flu*))

\section{Appendix 5. Search strategy - Epistemonikos, L*OVE List Coronavirus disease (COVID-19)}

by pico search: population: Covid-19; intervention: passive immunization

\section{Appendix 6. Search strategy Cochrane COVID-19 Study Register}

plasma OR hyperimmune OR hyper-immune OR IVIG OR immunoglobulin OR globulin OR gamma-globulin OR Y-Globulin OR hyper-Ig OR serum OR sera OR donor OR donation OR sero* OR flu-IVIG OR antiflu* OR anti-flu

\section{H I S T O R Y}

Protocol first published: Issue 12, 2020 


\section{CONTRIBUTIONS OF AUTHORS}

SJV: clinical expertise, and conception and writing of the protocol

VP: methodological expertise, and conception and writing of the protocol

CK: clinical and methodological expertise and advice

KLC: clinical expertise, and conception and writing of the review

IM: development of the search strategy

CD: development of the search strategy

EMW: clinical expertise and advice

AL: clinical expertise and advice

DJR: clinical expertise and advice

ZM: clinical expertise and advice

CS-O: clinical expertise and advice

LJE: clinical and methodological expertise, and conception and writing of the protocol

NS: methodological expertise and advice, and conception and writing of the protocol

\section{DECLARATIONS OF INTEREST}

SJV: has declared that they have no conflict of interest.

VP: has declared that they have no conflict of interest.

CK: has declared that they have no conflict of interest.

KLC: has declared that they have no conflict of interest.

IM: has declared that they have no conflict of interest.

CD: has declared that they have no conflict of interest.

EMW: has declared that they have no conflict of interest.

AL: I am actively involved in processing and managing the measurement of antibody activity within convalescent plasma (CP) and samples collected from patients in clinical trials designed to study outcomes of CP against COVID-19.

DJR: I am a consultant haematologist for NHS Blood and Transplant.

ZM: has declared that they have no conflict of interest.

CS-O: has declared that they have no conflict of interest.

LJE: I am a consultant haematologist for NHS Blood and Transplant.

NS: has declared that they have no conflict of interest.

\section{SOURCES OF SUPPORT}

\section{Internal sources}

- University Hospital of Cologne, Germany

Cochrane Cancer, Department I of Internal Medicine

- Sanquin Blood Supply, Netherlands

Center for Clinical Transfusion Research 
- Monash University, Australia

Transfusion Research Unit, Department of Epidemiology and Preventive Medicine

- NHS Blood and Transplant, UK

NHS Blood and Transplant

- Haematology Society of Australia and New Zealand, Australia

HSANZ and Leukaemia Foundation

\section{External sources}

- No sources of support supplied 